\title{
On some properties of $\gamma_{p}$-summing operators in Banach spaces
}

\author{
Yasuji Takahashi and Yoshiaki OKaZAKI \\ (Received October 7, 1985, Revised May 16, 1986)
}

\section{§ 1. Introduction}

The aim of this paper is to give new characterizations of Banach spaces which are of stable type $p$ and of $S Q_{p}$ type (or of $S_{p}$ type) by the properties of $\gamma_{p}$-summing operators $(0<p \leqq 2)$.

The $\gamma_{p}$-summing operator was introduced by Thang and Tien [23]. Let $E, F$ be Banach spaces and $0<p \leqq 2$. Denote by $L(E, F)$ the set of all bounded linear operators from $E$ into $F$. Then an operator $T$ in $L(E, F)$ is $\gamma_{p}$-summing if for each sequence $\left\{x_{n}\right\} \subset E$ with $\Sigma_{n}\left|<x_{n}, x^{\prime}>\right|^{p}<\infty$ for all $x^{\prime}$ $\in E^{\prime}$, the series $\Sigma_{n} T\left(x_{n}\right) \theta_{n}^{(p)}$ converges almost surely (a.s.) in $F$, where $\left\{\theta_{n}^{(p)}\right\}$ is a sequence of independent identically distributed real random variables with the characteristic function (ch.f.) $\exp \left(-|t|^{p}\right), t \in R$. Denote by $\Pi_{\gamma_{\rho}}(E, F)$ the set of all $\gamma_{p}$-summing operators from $E$ into $F$.

The $\gamma_{p}$-Radonifying operator was introduced by Linde, Mandrekar and Weron [6] as follows. Let $p$ be $1<p \leqq 2$ and $1 / p+1 / p^{\prime}=1$. Then an operator $T$ in $L\left(L_{p^{\prime}}, F\right)$ is $\gamma_{p}$-Radonifying if $\exp \left(-\left\|T^{\prime}\left(x^{\prime}\right)\right\|^{p}\right), x^{\prime} \in F^{\prime}$, is the ch. f. of a Radon measure on $F$, where $T^{\prime}$ is the adjoint of $T$. Denote by $\sum_{p}\left(L_{p^{\prime}}, F\right)$ the set of all $\gamma_{p}$-Radonifying operators from $L_{p^{\prime}}$ into $F$.

The classification problem of type $p$-stable Banach spaces, $1<p \leqq 2$, was studied by Chobanjan and Tarieladze [1], Kwapien [4], Linde, Mandrekar and Weron [6], Linde, Tarieladze and Chobanjan [7], Mandrekar and Weron [12], Mouchtari [15] and Thang and Tien [23]. The classifications by these authors are all based upon the properties of $\gamma_{p}$-Radonifying operators. In this paper, we shall adopt the $\gamma_{p}$-summing operators for such classifications. The reason why we use the $\gamma_{p}$-summing operators instead of $\gamma_{p}$-Radonifying operators will become clear through the discussions in Sections 3 and 4 . Here we point out that $\Pi_{\gamma_{p}}(E, F)$ has the so-called ideal property, that is, if $T \in \Pi_{\gamma_{\rho}}(E, F)$, then $\operatorname{TS} \in \Pi_{\gamma_{\rho}}(G, F)$ for every Banach space $G$ and every $S \in L(G, E)$. But in general, for $1<p<2, \Sigma_{p}\left(L_{p^{\prime}}, F\right)$ does not have the ideal property even in the case $G=L_{p^{\prime}}$. In the classification of type $p$-stable Banach spaces, $1<p \leqq 2$, Mandrekar and Weron 
[12] introduced the technical conditions $V_{p}($ i $)$ and $V_{p}$ (ii). From our considerations, these conditions are clarified as follows. The Banach space $E$ satisfies the condition $V_{p}(\mathrm{i})$ (resp. $V_{p}($ ii $)$ ) if and only if every $\gamma_{p^{-}}$ Radonifying operator $T: l_{p} \rightarrow E$ (resp. $L_{p^{\prime}}[0,1] \rightarrow E$ ) is $\gamma_{p^{-}}$-summing. That is to say, Mandrekar and Weron [12] required the property of $\gamma_{p}$-summing operators (the ideal property) explicitely, besides the $\gamma_{p}$-Radonifying operators.

In Sections 3 and 4 , we shall investigate the relationship between $\gamma_{p}$-summing operators and $p$-summing operators. The linear operator $T: E$ $\rightarrow F$ is said to be $p$-summing if for each sequence $\left\{x_{n}\right\} \subset E$ with $\Sigma_{n}\left|<x_{n}, x^{\prime}>\right|^{p}$ $<\infty$ for all $x^{\prime} \in E^{\prime}$, it holds that $\Sigma_{n}\left\|T\left(x_{n}\right)\right\|^{p}<\infty$. Denote by $\Pi_{p}(E, F)$ the set of all $p$-summing operators from $E$ into $F$. In [23], Thang and Tien proved that if $F$ dose not contain $c_{0}$, Then every $r$-summing operator $T: E$ $\rightarrow F$ is $\gamma_{p}$-summing, where $1<r<p<2$. We shall show that this result remains true without any additional assumption on $F$. Remark that this result is false in the case $r=p$. If $E$ is of stable type $p$, then it follows easily $\Pi_{\gamma_{p}}(G, E)=\Pi_{p}(G, E)$ for every Banach space $G$, as remarked by Thang and Tien [23]. We shall prove the converse: $\mathrm{E}$ is of stable type $p, 1<p<2$, if and only if for each (one infinite dimensional) space $L_{p^{\prime}}, \Pi_{\gamma_{p}}\left(L_{p^{\prime}}, E\right)=\Pi_{p}\left(L_{p^{\prime}}\right.$, $E)$. For the case $p=2$, it is well known that $\Pi_{\gamma_{2}}\left(L_{2}, E\right)=\Pi_{2}\left(L_{2}, E\right)$ if and only if $E$ is of stable cotype 2 (see Maurey [13]).

In Section 5, we shall give new characterizations of Banach spaces which are of stable type $p$ and of $S Q_{p}$ type, $1<p<2$, in terms of $\gamma_{p}$-summing operators. It is shown that $E$ is of stable type $p$ and of $S Q_{p}$ type, $1<p<2$, if and only if for each Banach space $G$, every dual $p$-nuclear operator $T: G$ $\rightarrow E$ is $\gamma_{p}$-summing. Here the term "each Banach space $G$ " can be reqlaced by " one infinite dimensional space $L_{p^{\prime}}$ ". By a result of Mouchtari [15], we can conclude that $E$ is of stable type $p, 1<p \leqq 2$, if and only if for each (one infinite dimensional) space $L_{p^{\prime}}$, every dual $p$-decomposable operator $T: L_{p^{\prime}}$ $\rightarrow E$ is $\gamma_{p}$-Radonifying. Here if we replace " $\gamma_{p}$-Radonifying" by " $\gamma_{p}$ summing ", then we have more rich result as follows: $E$ is of stable type $p$ and of $S Q_{p}$ type, $1<p<2$, if and only if for each (one infinite dimensional) space $L_{p^{\prime}}$, every dual $p$-decomposable operator $T: L_{p} \rightarrow E$ is $\gamma_{p^{-}}$-summing. This result depends on the ideal property of $\Pi_{\gamma_{p}}\left(L_{p^{\prime}}, E\right)$.

In section 6 , we shall characterize Banach spaces which are of stable type $p$ and of $S_{p}$ type, $1<p<2$, in terms of $\gamma_{p}$-summing operators and the other operator ideals in the sense of Persson and Pietsch [17]. It is shown that $E$ is of stable type $p$ and of $S_{p}$ type, $1<p<2$, if and only if for each Banach space $G$, every dual quasi-p-nuclear operator $T: G \rightarrow E$ is 
$\gamma_{p^{-}}$-summing, and, if and only if for each (one infinite dimensional) space $L_{p^{\prime}}$, every dual quasi- $p$-nuclear operator $T: L_{p^{\prime}} \rightarrow E$ is $\gamma_{p^{-}}$-summing. This result remains true in the case where "quasi- $p$-nuclear" is replaced by " $p$-summing". Now we refer to the relation of our last result and the works of Linde, Mandrekar and Weron [6] and Thang and Tien [23]. Linde, Mandrekar and Weron [6] proved that $E$ is of stable type $p$ and of $S_{p}$ type, $1<p<2$, if and only if for each (one infinite dimensional) separable space $L_{p^{\prime}}$, every dual $p$-summing operator $T: L_{p} \rightarrow E$ is $\gamma_{p}$-Radonifying. The case $L_{p^{\prime}}=l_{p^{\prime}}$ is due to Thang and Tien [23]. We shall remark that only in the case $L_{p^{\prime}}=l_{p^{\prime}}$, our result is in fact equivalent to those of Linde, Mandrekar and Weron [6] and Thang and Tien [23].

Throughout the paper, we assume that all linear spaces are with real coefficients.

\section{$\S 2$. Notations and definitions}

By $E$ we denote a Banach space with the dual $E^{\prime}$. A sequence $\left\{x_{n}\right\}$ in $E$ is said to be weakly $p$-summable, $0<p<\infty$, if it holds that $\Sigma_{n}\left|<x_{n}, x^{\prime}>\right|^{p}$ $<\infty$ for all $x^{\prime} \in E^{\prime}$. Let $F$ be another Banach space. As mentioned in Section 1 , an operator $T$ in $L(E, F)$ is $p$-summing if for each weakly $p$-summable sequence $\left\{x_{n}\right\}$ in $E$, we have $\Sigma_{n}\left\|T\left(x_{n}\right)\right\|^{p}<\infty$; and in particular, if $T$ is $p$-summing for all $p>0$, then $T$ is said to be completely summing. Let $p$ be $1 \leqq p<\infty$ and $1 / p+1 / p^{\prime}=1$. We say that an operator $T$ in $L(E, F)$ is quasi-p-unclear if there exists a sequcnce $\left\{x_{n}^{\prime}\right\}$ in $E^{\prime}$ with $\Sigma_{n}$ $\left\|x_{n}^{\prime}\right\|^{p}<\infty$ such that $\|T(x)\| \leqq\left(\sum_{n}\left|<x, x_{n}^{\prime}>\right|^{p}\right)^{1 / p}$ for all $x \in E ; T$ is $p$-nuclear if $T$ is factorized by the bounded linear operators $V: E \rightarrow l_{\infty}, D: l_{\infty} \rightarrow l_{p}$ and $W: l_{p} \rightarrow F$, where $D=\left(\alpha_{n}\right)$ is a diagonal operator with $\sum_{n}\left|\alpha_{n}\right|^{p}<\infty$; and $T$ is of type $N^{p}$ if $T$ is factorized by the bounded linear operators $V: E \rightarrow l_{p^{\prime}}$, $D: l_{p} \rightarrow l_{1}$ and $W: l_{1} \rightarrow F$, where $D$ is of the same kind as above. The set of all $p$-nuclear operators (resp. operators of type $N^{p}$ ) from $E$ into $F$ will be denoted by $N_{p}(E, F)$ (resp. $N^{p}(E, F)$ ). For the details of these operators, we refer to [16], [17] and [18].

Now let $(\Omega, \Sigma, \mu)$ be a probability space and $1 \leqq p<\infty$. As usual, by $L_{p}(\Omega, \mu, E)$, or $L_{p}(E)$, we denote a Banach space of all $\mu$-measurable $E$-valued functions on $\Omega$ which are strongly $p$-integrable. If $E=R$ (real line), then we write $L_{p}(\mu)$, or $L_{p}$, instead of $L_{p}(\Omega, \mu, R)$. We say that an operator $T$ in $L\left(E^{\prime}, L_{p}\right)$ is $p$-decomposable if there exists a function $\psi$ in $L_{p}$ $(\Omega, \mu, E)$ such that $T\left(x^{\prime}\right)=\left\langle\psi(\bullet), x^{\prime}\right\rangle$ for all $x^{\prime} \in E^{\prime}$. Kwapien [2] proved that every $p$-summing operator from $L_{p^{\prime}}$ into $E$ is dual $p$-decomposable $(1<p<\infty)$, and in particular, if $E$ is reflexive, then every operator of type $N^{p}$ from $E^{\prime}$ into $L_{p}$ is $p$-decomposable (see Persson [16]). 
Persson [16] also proved that every $p$-decomposable operator from $E^{\prime}$ into $L_{p}$ is $p$-nuclear.

Following Kwapien [3], we say that $E$ is of $S_{p}$ type (resp. $S Q_{p}$ type) if $E$ is isomorphic to a subspace (resp. a subspace of a quotient) of some $L_{p}$.

For $0<p \leqq 2$, we say that $E$ is of stable type $p$ if for each sequence $\left\{x_{n}\right\}$ in $E, \sum_{n}\left\|x_{n}\right\|^{p}<\infty$ implies the series $\sum_{n} x_{n} \theta_{n}^{(p)}$ converges a.s., and $E$ is of stable cotype $p$, if, conversely the a. s. convergence of $\sum_{n} x_{n} \theta_{n}^{(p)}$ implies $\sum_{n}\left\|x_{n}\right\|^{p}<\infty$. It is well known that every Banach space is of stable type $p$ with $p<1$ and of stable cotype $p$ with $p<2$ (see Maurey [13]).

\section{$\S 3$. $\gamma_{p}$-summing operators and $\gamma_{p}$-Radonifying operators}

In this section, we investigate the properties of $\gamma_{p}$-summing operators comparing with $\gamma_{p}$-Radonifying operators. Let $p$ be $1<p \leqq 2$ and $1 / p+1 / p^{\prime}=$ 1. Denote by $\left\{e_{n}\right\}$ the sequence of canonical unit vectors in $l_{p^{\prime}}$. It is known that a linear operator $T: l_{p} \rightarrow E$ is $\gamma_{p}$-Radonifying if and only if the series $\Sigma_{n}$ $T\left(e_{n}\right) \theta_{n}^{(p)}$ converges a.s. in $E$ (see [6], Corollary 1 or [23], Lemma 1). Since the sequence $\left\{e_{n}\right\}$ is weakly $p$-summable, it follows that every $\gamma_{p^{-}}$ summing operator from $l_{p^{\prime}}$ into $E$ is $\gamma_{p}$-Radonifying. But in general, the converse is not true. Of course for the case $p=2$, every $\gamma_{2}$-Radonifying operator from $l_{2}$ into $E$ is $\gamma_{2}$-summing (see [23], Theorem 3).

First we prepare the following elementary lemma.

LEMMA 3.1. Let $T$ be a bounded linear operator from a Banach space $E$ into a Banach space $F$. Then for $1<p \leqq 2$, the following are equivalent.

(1) $T$ is $\gamma_{p}$-summing.

(2) For each $S \in L\left(l_{p^{\prime}}, E\right)$, TS is $\gamma_{p}$-Radonifying.

PROOF. The assertion follows from the fact that for each weakly $p$-summable sequence $\left\{x_{n}\right\}$ in $E$, there exists an operator $S$ in $L\left(l_{p^{\prime}}, E\right)$ such that $S\left(e_{n}\right)=x_{n}$ for all $n$.

Let $E$ be a Banach space and $T$ be a bounded linear operator from $E^{\prime}$ into $L_{p}, 1<p \leqq 2$. Denote by $N(T)$ the cylindrical measure on $E$ with the ch. f. $\exp \left(-\|T(x)\|^{p}\right), x^{\prime} \in E^{\prime}$. We say that $N(T)$ is Radon if $N(T)$ is a Radon measure on $E$. For $1<p<2$, it is easy to see that for an operator $T$ in $L\left(E^{\prime}, l_{p}\right), N(T)$ is Radon implies $T$ is $p$-decomposable. In the case where $(\Omega, \mu)$ is a probability space and $1<p<2$, Linde [8] proved that for an operator $T$ in $L\left(E^{\prime}, L_{p}(\mu)\right), N(T)$ is Radon implies $T$ is $p$-decomposable, i. e. there exists a function $\psi$ in $L_{p}(\Omega, \mu, E)$ such that $T(x)=<$ $\psi(\bullet), x^{\prime}>$ for all $x^{\prime} \in E^{\prime}$. In this case, if we define an operator $S$ in $L\left(L_{p^{\prime}}\right.$, $E$ ) by $S(f)=\int \psi(\boldsymbol{\omega}) f(\boldsymbol{\omega}) d \mu(\boldsymbol{\omega})$ for all $f \in L_{p^{\prime}}$, then $S$ is $\gamma_{p}$-Radonifying 
and $T=S^{\prime}$.

Let $E$ be a Banach space and $1<p \leqq 2$. Following Mandrekar and Weron [12], we say that $E$ satisfies the condition $V_{p}(\mathrm{i})$ if for each $T \in L$ $\left(E^{\prime}, l_{p}\right), N(T)$ is Radon implies $N(S T)$ is Radon for all $S \in L\left(l_{p}, l_{p}\right)$; and $E$ satisfies the condition $V_{p}($ ii $)$ if for each $T \in L\left(E^{\prime}, L_{p}[0,1]\right), N(T)$ is Radon implies $N(S T)$ is Radon for all $S \in L\left(L_{p}[0,1], l_{p}\right)$. Of course every Banach space satisfies the conditions $\mathrm{V}_{2}(\mathrm{i})$ and $\mathrm{V}_{2}$ (ii). Then from the observations above and Lemma 3.1, we have the following.

Corollary 3.2. For $1<p \leqq 2$, the following properties of a Banach space are equivalent.

(1) E satisfies the condition $V_{p}(\mathrm{i})$.

(2) Every $\gamma_{p}$-Radonifying operator from $l_{p^{\prime}}$ into $E$ is $\gamma_{p}$-summing.

CoROllary 3.3. For $1<p \leqq 2$, the following properties of a Banach space $E$ are equivalent.

(1) E satisfies the condition $V_{p}$ (ii).

(2) Every $\gamma_{p}$-Radonifying operator from $L_{p}[0,1]$ into $E$ is $\gamma_{p}$-summing.

Now we shall investigate the relationship between $\gamma_{p}$-summing and $p$-summing operators. It is clear that for $0<p<1$, every $p$-summing operator is $\gamma_{p}$-summing because every Banach space is of stable type $p$ with $p<1$, and on the other hand, for $0<p<2$, every $\gamma_{p}$-summing operator is $p$-summing because every Banach space is of stable cotype $p$ with $p<2$ (see [13]).

THEOREM 3.4. Let $1<r<p<2$. Then every $r$-summing operator from a Banach space $E$ into a Banach space $F$ is $\gamma_{p}$-summing.

Proof. Let $T$ be an $r$-summing operator from $E$ into $F$. If $S \in L\left(l_{p^{\prime}}, E\right)$, then $T S$ is $r$-summing. Using Schwartz theorem [19] it follows that TS is $r$-Radonifying. Denote by $\gamma_{p}$ the canonical $p$-stable cylindrical measure on $l_{p^{\prime}}$ with the ch. f. $\exp \left(-\|x\|^{p}\right), x \in l_{p}$. Since the cylindrical measure $\gamma_{p}$ is of type $r$ with $r<p, T S\left(\gamma_{p}\right)$ extends to a Radon measure on $E$, that is, $T S$ is $\gamma_{p}$-Radonifying. Thus the assertion follows from Lemma 3.1.

REMARK. Theorem 3.4 was proved by Thang and Tien [23] under the assumption that $F$ does not contain $c_{0}$. For the case $p=2$, every $r$-summing operator is $\gamma_{2}$-summing because the cylindrical measure $\gamma_{2}$ on $l_{2}$ is of type $r$ with $0<r<\infty$.

Let us recall that a Banach space $\mathrm{E}$ is $p$-Pietsch, $0<p<\infty$, provided that every $p$-summing operator from $E$ into a Banach space $F$ is completely summing. It is known (cf. [20]) that if $E$ is of infinite dimension, then $E$ 
is not $(2+\varepsilon)-$ Pietsch, $\varepsilon>0$, on the other hand, every Banach space is $p$-Pietsch with $0<p<1$. If $E^{\prime}$ is of stable type $p, 1 \leqq p \leqq 2$, then every factor space of $E$ is $p$-Pietsch, and in particular, $L_{r^{\prime}}$ and its factor space are $p$-Pietsch, $0<p<r \leqq 2$. For the details we refer to [14] and [20].

Corollary 3.5. Suppose that $E$ is p-Pietsch, $1<p<2$. Then every p-summing operator from $E$ into a Banach space $F$ is $\gamma_{p}$-summing.

THEOREM 3. 6. Let $1<p<2$. Then the following properties of a Banach space $E$ are equivalent.

(1) $E$ is p-Pietsch.

(2) There is an $r$ with $p<r<2$ such that every $\gamma_{r}$-summing operator from $E$ into a Banach space $F$ is completely summing.

Proof. Suppose that $E$ is $p$-Pietsch. Then there is an $r$ with $p<r<$ 2 such that $E$ is $r$-Pietsch (see [20], 16.1. Theorem). Since every $\gamma_{r}$-summing operator is $r$-summing with $r<2$, (2) holds. Conversely, suppose that (2) holds. If $T$ is $p$-summing operator from $E$ into a Banach space $F$, then by Theorem 3.4, $T$ is $\gamma_{r}$-summing with $p<r<2$. From the assumption (2), it fomlows that $T$ is completely summing. Thus $E$ is $p$-Pietsch.

This completes the proof.

\section{$\S 4$. Banach spaces of stable type $p$}

In this section, we characterize the type $p$-stable Banach space $E$ by $\Pi_{\gamma_{p}}$ $\left(L_{p^{\prime}}, E\right)=\Pi_{p}\left(L_{p^{\prime}}, E\right)$, where $L_{p^{\prime}}$ is of infinite dimension $(1<p<2$ and $1 / p+1 /$ $\left.p^{\prime}=1\right)$. In Section 3, we proved that if a Banach space $G$ is $p$-Pietsch, $1<$ $p<2$, then $\Pi_{\gamma_{\rho}}(G, E)=\Pi_{p}(G, E)$ for every Banach space $E$. The following theorem says that this result is not true even in the case where $G$ is $(p-\varepsilon)$ -Pietsch for all $\varepsilon>0$.

THEOREM 4.1. Let $1<p<2$. Then the following properties of a Banach space $E$ are equivalent.

(1) $E$ is of stable type $p$.

(2) For each Banach space $G$, there holds $\Pi_{\gamma_{p}}(G, E)=\Pi_{p}(G, E)$.

(3) For each (one infinite dimensional) space $L_{p^{\prime}}$, there holds $\Pi_{\gamma_{\phi}}\left(L_{p^{\prime}}\right.$, $E)=\Pi_{p}\left(L_{p^{\prime}}, E\right)$.

Proof. The implications $(1) \Longrightarrow(2) \Longrightarrow(3)$ are clear. We show that $(3) \Longrightarrow(1)$ holds. Suppose that for some infinite dimensional space $L_{p^{\prime}}$, The inclusion $\Pi_{p}\left(L_{p^{\prime}}, E\right) \subset \Pi_{\gamma_{p}}\left(L_{p^{\prime}}, E\right)$ holds. Since $L_{p}$ is of infinite dimension, $L_{p}$ contains a complemented subspace which is linearly isometric 
to $l_{p}$ (cf. [11]). Hence the identity map : $l_{p} \rightarrow l_{p}$ is factorized by the bounded linear operators $J: l_{p} \rightarrow L_{p}$ and $P: L_{p} \rightarrow l_{p}$, where $J$ is an isometric imbedding. To prove (1), let $T$ be a $p$-summing operator from $l_{p^{\prime}}$ into $E$. Then $T J^{\prime}: L_{p^{\prime}}$ $\rightarrow E$ is $p$-summing. From the assumption (3) it follows that $T J^{\prime}$ is $\gamma_{p^{-}}$ summing and so is $T=T J^{\prime} P^{\prime}$ (the ideal property). Thus the assertion follows from [22], Proposition 3.

Remark. As is shown in this proof, if a Banach space $G$ contains a complemented subspace which is linearly isometric to $l_{p^{\prime}}$, then $E$ is of stable type $p, 1<p<2$, if and only if $\Pi_{\gamma_{p}}(G, E)=\Pi_{p}(G, E)$. For the case $p=1$, it can be shown that $E$ is of stable type 1 if and only if $\Pi_{\gamma_{1}}\left(c_{0}, E\right)=\Pi_{1}\left(c_{0}, E\right)$. On the other hand, Theorem 4.1 is false in the case $p=2$. In fact, it is well known that $E$ has one of the properties (2) and (3) for $p=2$ if and only if $E$ is of stable cotype 2 (see [13]).

Corollary 4.2. Suppose that $L_{p}$ is of infinite dimension, $1<p<2$. If $E$ is not of stable type $p$, then there are p-summing operators from $L_{p^{\prime}}$ into $E$ which are not $\gamma_{p}$-summing. In particular, $L_{p^{\prime}}$ is not p-Pietsch.

For the case $p=2$, it is well known (cf. [1], [7]) that a Banach space $E$ is of stable type 2 if and only if for each $T \in L(H, E), T^{\prime}$ is 2-summing implies $T$ is $\gamma_{2}$-Radonifying, where $H$ is an infinite dimensional Hilbert space. The following theorem says that this result remains true in the case where "2-summing" is replaced by "2-nuclear". Remark that every 2-nuclear operator is 2-summing, but in general, the converse is not true.

THEOREM 4.3. The following properties of a Banach space $E$ are equivalent.

(1) $E$ is of stable type 2.

(2) For each $T \in L(H, E), T^{\prime}$ is 2-nuclear implies $T$ is $\gamma_{2}$-summing.

(3) $N^{2}(H, E) \subset \Pi_{\gamma_{2}}(H, E)$.

Proof. Suppose that (1) holds. To prove (2), by Lemma 3. 1, it is enough to show that for each $T \in L\left(l_{2}, E\right), T^{\prime}$ is 2-nuclear implies $T$ is $\gamma_{2}$-Radonifying. Let $T: l_{2} \rightarrow E$ be a bounded linear operator such that $T^{\prime}$ is 2-nuclear. Then $T^{\prime}$ is factorized by the bounded linear operators $V: E^{\prime} \rightarrow l_{\infty}, D: l_{\infty} \rightarrow l_{2}$ and $W: l_{2} \rightarrow l_{2}$, where $D=\left(\alpha_{n}\right)$ is a diagonal operator with $\sum_{n}\left|\alpha_{n}\right|^{2}<\infty$. If we put $S=D V$, then $S^{\prime}$ is a bounded linear operator from $l_{2}$ into $E^{\prime \prime}$ such that $\sum_{n}\left\|S^{\prime}\left(e_{n}\right)\right\|^{2}<\infty$, where $e_{n}$ denotes the $n$-th unit vector in $l_{2}$. Since $E^{\prime \prime}$ is of stable type 2, the series $\sum_{n} S^{\prime}\left(e_{n}\right) \theta_{n}^{(2)}$ converges a. $\mathrm{s}$. in $E^{\prime \prime}$, that is, $S^{\prime}$ is $\gamma_{2}$-Radonifying and so is $T^{\prime \prime}=S^{\prime} W^{\prime}$ (see [23]). Since $E$ does not contain $c_{0}$, it follows that $T$ 
$: l_{2} \rightarrow E$ is $\gamma_{2}$-Radonifying (see [7]). Thus (2) holds. On the other hand, $(2) \Longrightarrow(3)$ is clear. Now suppose that (3) holds. To prove (1), let $\left\{x_{n}\right\}$ be a sequence in $E$ such that $\sum_{n}\left\|x_{n}\right\|^{2}<\infty$. Then there is an operator $T$ in $L\left(l_{2}, E\right)$ such that $T\left(e_{n}\right)=x_{n}$ for all $n$. Evidently, $T$ is an operator of type $N^{2}$. Let $J$ be an isometric imbedding from $l_{2}$ into $H$. Since $T J^{\prime}: H \rightarrow E$ is of type $N^{2}$, by the assumption (3), it follows that $T J^{\prime}$ is $\gamma_{2}$-summing and so is $T$. Thus the series $\sum_{n} x_{n} \theta_{n}^{(2)}=\sum_{n} T\left(e_{n}\right) \theta_{n}^{(2)}$ converges a.s. in $E$ proving (1).

This completes the proof.

REMARK. As is shown in this proof, the property (2) is equivalent to the fact that for each Banach space $G$ and each $T \in L(G, E), T^{\prime}$ is 2-nuclear implies $T$ is $\gamma_{2}$-summing. We note here that if $H$ is a separable Hilbert space, then for each Banach space $E$, every $\gamma_{2}$-summing operator from $H$ into $E$ is of type $N^{2}$ (see [5]).

\section{$\S 5$. Banach spaces of stable type $p$ and of $S Q_{p}$ type}

In this section, we shall give new characterizations of Banach spaces which are of stable type $p$ and of $S Q_{p}$ type, $1<p<2$, in terms of $\gamma_{p}$-summing operators. Throughout this section, let $E$ be a Banach space, $(\Omega, \mu)$ be a probability space and $1<p<2$. As mentioned in Section 2 , an operator $T$ in $L\left(E^{\prime}, L_{p}(\mu)\right)$ is $p$-decomposable if there exists a function $\psi$ in $L_{p}(\Omega, \mu, E)$ such that $T(x)=\left\langle\psi(\cdot), x^{\prime}\right\rangle$ for all $x^{\prime} \in E^{\prime}$. Now suppose that $L_{p}(\mu)$ is of infinite dimension. Then there exists an isometric imbedding $J$ from $l_{p}$ into $L_{p}$ (cf. [11]]). First we establish the following elementary lemma.

LEMmA 5.1. Let $T$ be a bounded linear operator from $l_{p^{\prime}}, 1<p<2$, into $E$. Then the following are equivalent.

(1) $\sum_{n}\left\|T\left(e_{n}\right)\right\|^{p}<\infty$.

(2) $J T^{\prime}: E^{\prime} \rightarrow L_{p}(\mu)$ is p-decomposable.

Proof. Suppose that (1) holds. Let $I=\left\{n ; T\left(e_{n}\right) \neq 0\right\}$. If $I$ is a finite set, then (2) clearly holds. If $I$ is an infinite set, then we take a positive constant $C$ such that $C=\left(\Sigma_{n}\left\|T\left(e_{n}\right)\right\|^{p}\right)^{-1 / p}$ and define a Radon probability measure $\nu$ on $E$ by $\nu=\sum_{n \in I} \alpha_{n} \delta_{x_{n}}$, where $\alpha_{n}=C^{p}\left\|T\left(e_{n}\right)\right\|^{p}, x_{n}=T$ $\left(e_{n}\right) / C\left\|T\left(e_{n}\right)\right\|$ and $\delta_{x_{n}}$ is a Dirac measure of the point $x_{n}$. Then we have

$$
\left\|J T^{\prime}\left(x^{\prime}\right)\right\|^{p}=\left\|T^{\prime}(x)\right\|^{p}=\int_{E}\left|<x, x^{\prime}>\right|^{p} d \nu(x), x^{\prime} \in E^{\prime},
$$

and 


$$
\int_{E}\|x\|^{p} d \nu(x)=\Sigma_{n}\left\|T\left(e_{n}\right)\right\|^{p}<\infty .
$$

Using Linde [9], Theorem 4, it follows that $J T^{\prime}$ is $p$-decomposable. Thus (2) holds. On the other hand, suppose that (2) holds. Then there is a function $\psi$ in $L p(\Omega, \mu, E)$ such that $J T^{\prime}(x)=\left\langle\psi(\bullet), x^{\prime}\right\rangle$ for all $x^{\prime} \in E^{\prime}$. If we put $\sigma=\psi(\mu)$, then $\sigma$ is a Radon probability measure on $E$ and there holds

$$
\left\|T^{\prime}\left(x^{\prime}\right)\right\|^{p}=\left\|J T^{\prime}\left(x^{\prime}\right)\right\|^{p}=\left.\int_{E}\left|<x, x^{\prime}\right\rangle\right|^{p} d \sigma(x), x^{\prime} \in E^{\prime},
$$

and

$$
\int_{E}\|x\|^{p} d \sigma(x)<\infty
$$

Let $\left\{\alpha_{n}\right\}$ be a sequence of positive real numbers such that $\sum_{n} \alpha_{n}=1$. Define a Radon probability measure $\nu$ on E by $\nu=\sum_{n} \alpha_{n} \delta_{x_{n}}$, where $x_{n}=T\left(e_{n}\right) /\left(\alpha_{n}\right)^{1 / p}$. Then

$$
\int_{E}\left|<x, x^{\prime}>\right|^{p} d \nu(x)=\int_{E}\left|<x, x^{\prime}>\right|^{p} d \sigma(x), x^{\prime} \in E^{\prime} .
$$

Hence by Linde [9], Theorem 4 , we have

$$
\sum_{n}\left\|T\left(e_{n}\right)\right\|^{p}=\int_{E}\|x\|^{p} d \nu(x)<\infty .
$$

Thus (1) holds, and the proof is completed.

Proposition 5.2. Let $1<p<2$. Then every $\gamma_{p}$-summing operator from $L_{p}(\mu)$ into a Banach space $E$ is dual p-decomposable.

Proof. The assertion easily follows from Kwapien [2], Theorem 2 and the fact that every $\gamma_{p}$-summing operator is $p$-summing with $p<2$.

COROLLARY 5.3. Let $1<p<2$. Then every $\gamma_{p}$-summing operator from $L_{p}(\mu)$ into $E$ is of type $N^{p}$, and in particular, dual p-nuclear.

Proof. The assertion follows from Proposition 5.2 and Persson [16], Theorem 2.

THEORER 5.4. Let $1<p<2$. Then the following properties of a Banach space $E$ are equivalent.

(1) $E$ is of stable type $p$ and of $S Q_{p}$ type.

(2) For each Banach space $G$ and each $T \in L(G, E), T^{\prime}$ is p-nuclear implies $T$ is $\gamma_{p}$-summing.

(3) For each Banach space $G$, the inclusion $N^{p}(G, E) \subset \Pi_{\gamma_{p}}(G, E)$ 
holds.

(4) For each (one infinite dimensional) space $L_{p^{\prime}}(\mu), T \in \Pi_{\gamma_{\rho}}\left(L_{p^{\prime}}, E\right)$ if and only if $T^{\prime} \in N_{p}\left(E^{\prime}, L_{p}\right)$.

(5) For each (one infinite dimensional) space $L_{p}(\mu)$, there holds $N^{p}$ $\left(L_{p^{\prime}}, E\right)=\Pi_{\gamma_{p}}\left(L_{p^{\prime}}, E\right)$.

(6) For each (one infinite dimensional) space $L_{p^{\prime}}(\mu), T \in \Pi_{\gamma_{p}}\left(L_{p^{\prime}}, E\right)$ if and only if $T^{\prime}$ is p-decomposable.

To prove this theorem, we need the following

Lemma 5.5 (cf. [21]). Let $1<p<\infty$. Then the following properties of a Banach space $E$ are equivalent.

(1) $E$ is of $S Q_{p}$ type.

(2) $E^{\prime}$ is of $S Q_{p^{\prime}}$ type.

(3) For each $T \in L\left(l_{p^{\prime}}, E\right), \Sigma_{n}\left\|T\left(e_{n}\right)\right\|^{p}<\infty$ implies $T$ is p-summing.

Proof of Theorem 5.4. Suppose that (1) holds. To prove (2), let $G$ be any Banach space and $T$ be an operator in $L(G, E)$ such that $T^{\prime}$ is $p$-nuclear. Since $E$ is of $S Q_{p}$ type, by Lemma 5.5, $E^{\prime}$ is of $S Q_{p^{\prime}}$ type. Using Kwapien [3], Corollary 8, it follows that $T$ is $p$-summing. But this implies that $T$ is $\gamma_{p}$-summing because $E$ is of stable type $p$. Thus (2) holds. Since every operator of type $N^{p}$ is clearly dual $p$-nuclear, (2) implies (3). On the other hand, $(2) \Longrightarrow(4)$ and $(3) \Longrightarrow(5)$ follow from Corollary 5.3, and (5) $\Longrightarrow(6)$ follows from Proposition 5. 2 and Persson [16], Theorem 2. It remains to prove that (6) implies (1). Suppose that (6) holds. Since $L_{p}$ $(\mu)$ is of infinite dimension, it contains a complemented subspace which is linearly isometric to $l_{p}$ (see [11]). Hence the identity map: $l_{p} \rightarrow l_{p}$ is factorized by the bounded linear operators $J: l_{p} \rightarrow L_{p}$ and $P: L_{p} \rightarrow l_{p}$, where $J$ is an isometric imbedding. Now let $T$ be a bounded linear operator from $l_{p^{\prime}}$ into $\mathrm{E}$ such that $\Sigma_{n}\left\|T\left(e_{n}\right)\right\|^{p}<\infty$. Then by Lemma 5.1, $J T^{\prime}$ is $p$-decomposable. Hence it follows from the assumption (6) that $T J^{\prime}$ is $\gamma_{p}$-summing, and so is $T=T J^{\prime} P^{\prime}$ (the ideal property). This implies that $E$ is of stable type $p$ (see Theorem 4.1). Here we remark that every $\gamma_{p}$ summing operator is $p$-summing with $p<2$. Thus by Lemma 5.5, it follows that $E$ is of $S Q_{p}$ type, proving (1).

This completes the proof.

REMARK. In Theorem 5.4, we shall consider the case $p=2$. Then it can be shown in this case that $E$ has the property (6) if and only if $E$ is isomorphic to a Hilbert space. In fact, if $E$ has the property (6) for $p=2$, then it is easy to see that $E$ is of stable type 2 and of stable cotype 2. But this means that $E$ is isomorphic to a Hilbert space (see Kwapien [4]). On 
the other hand, $E$ has one of the properties (2), (3), (4) and (5) for $p=2$ if and only if $E$ is of stable type 2 (see Theorem 4.3).

\section{$\S 6$. Banach spaces of stable type $p$ and of $S_{p}$ type}

In this section, we characterize Banach spaces which are of stable type $p$ and of $S_{p}$ type, $1<p<2$, in terms of $\gamma_{p}$-summing operators. The following theorem is an analogue of a result due to Lindenstrauss and Pelczyński [10] which characterizes subspaces of $L_{p}$.

THEOREM 6.1. Let $1<p<2$. Then the following properties of a Banach space $E$ are equivalent.

(1) $E$ is of stable type $p$ and of $S_{p}$ type.

(2) If $\left\{x_{n}\right\}$ and $\left\{y_{n}\right\}$ are two sequences in $E$ such that

$$
\Sigma_{n}\left|<y_{n}, x^{\prime}>\right|^{p} \leqq \Sigma_{n}\left|<x_{n}, x^{\prime}>\right|^{p} \text { for all } x^{\prime} \in E^{\prime} \text {, }
$$

and $\sum_{n}\left\|x_{n}\right\|^{p}<\infty$, then the series $\sum_{n} y_{n} \theta_{n}^{(p)}$ converges a. s. in $E$.

PROOF. Since every Banach space is of stable cotype $p$ with $p<2$ (cf. [13]), the assertion follows from the criterion of Lindenstrauss-Pelczyński [10] on imbedding of a Banach space into $L_{p}$.

REMARK. For the case $p=2$, it can be easily shown that $E$ has the property (2) for $p=2$ if and only if $E$ is of stable type 2 .

The following result is due to Thang and Tien [23].

Corollary 6.2. Let $1<p<2$. Then the following properties of a Banach space $E$ are equivalent.

(1) $E$ is of stable type $p$ and of $S_{p}$ type.

(2) For each $T \in L\left(l_{p^{\prime}}, E\right), T$ is $\gamma_{p}$-Radonifying if and only if $T^{\prime}$ is p-summing.

Proof. (1) $\Longrightarrow(2)$ easily follows from Kwapien [3], Corollary 6 . Conversely, suppose that (2) holds. To prove (1), take two sequences $\left\{x_{n}\right\}$ and $\left\{y_{n}\right\}$ in $E$ as in (2) of Theorem 6.1. Then there is an operator $T$ in $L\left(l_{p^{\prime}}, E\right)$ such that $T\left(e_{n}\right)=y_{n}$ for all $n$. Evidently, $T^{\prime}$ is $p$-summing. From the assumption (2), it follows that $T$ is $\gamma_{p}$-Radonifying, that is, the series $\sum_{n} y_{n} \theta_{n}^{(p)}=\sum_{n} T\left(e_{n}\right) \theta_{n}^{(p)}$ converges a. s. in $E$ (see [6] or [23]). Thus the assertion follows from Theorem 6.1.

Theorem 6.3. Let $1<p<2$. Then the following properties of a Banach space $E$ are equivalent.

(1) $E$ is of stable type $p$ and of $S_{p}$ type.

(2) For each Banach space $G$ and each $T \in L(G, E), T^{\prime}$ is p-summing 
implies $T$ is $\gamma_{p}$-summing.

(3) For each (one infinite dimensional) space $L_{p^{\prime}}(\mu), T \in \Pi_{\gamma_{p}}\left(L_{p^{\prime}}, E\right)$ if and only if $T^{\prime}$ is quasi-p-nuclear.

(4) For each (one infinite dimensional) space $L_{p^{\prime}}(\mu)$, if $S$ and $T$ are bounded linear operators from $L_{p^{\prime}}$ into $E$ such that

$$
\left\|S^{\prime}\left(x^{\prime}\right)\right\| \leqq\left\|T^{\prime}\left(x^{\prime}\right)\right\| \text { for all } x^{\prime} \in E^{\prime},
$$

then $T^{\prime}$ is p-decomposable implies $S$ is $\gamma_{p}$-summing.

ProOF. (1) $\Longrightarrow(2)$ follows from Kwapien [3], Corollary 6. On the other hand, $(2) \Longrightarrow(3)$ follows from Corollary 5.3, and $(2) \Longrightarrow(4)$ easily follows from Persson [16], Theorem 1. It remains to prove that $(3) \Longrightarrow(1)$ and $(4) \Longrightarrow(1)$ hold. Let $\left\{x_{n}\right\}$ and $\left\{y_{n}\right\}$ be two sequences in $E$ such that

$$
\Sigma_{n}\left|<y_{n}, x^{\prime}>\|^{p} \leqq \Sigma_{n}\right|<x_{n}, x^{\prime}>\left.\right|^{p} \text { for all } x^{\prime} \in E^{\prime}
$$

and

$$
\sum_{n}\left\|x_{n}\right\|^{p}<\infty \text {. }
$$

Then there are operators $S$ and $T$ in $L\left(l_{p^{\prime}}, E\right)$ such that $S\left(e_{n}\right)=y_{n}$ and $T$ $\left(e_{n}\right)=x_{n}$ for all $n$. Evidently, $S^{\prime}$ is quasi- $p$-nuclear, and there holds

$$
\left\|S^{\prime}\left(x^{\prime}\right)\right\| \leqq\left\|T^{\prime}\left(x^{\prime}\right)\right\| \text { for all } x^{\prime} \in E^{\prime} .
$$

Now suppose that (3) holds. Since $L_{p}(\mu)$ is of infinite dimension, it contains a complemented subspace which is linearly isometric to $l_{p}$ (cf. [11]). Hence the identity map : $l_{p} \rightarrow l_{p}$ is factorized by the bounded linear operators $J: l_{p} \rightarrow L_{p}$ and $P: L_{p} \rightarrow l_{p}$, where $J$ is an isometric imbedding. Since $J S^{\prime}: E^{\prime}$ $\rightarrow L_{p}$ is quasi-p-nuclear, from the assumption (3) it follows that $S J^{\prime}$ is $\gamma_{p^{-}}$ summing and so is $S=S J^{\prime} P^{\prime}$. Thus the series $\sum_{n} y_{n} \theta_{n}^{(p)}$ converges a. s. in $E$, and (1) holds by Theorem 6.1. Finally, suppose that (4) holds. Let $J$ and $P$ be as above. Then by Lemma 5.1, $J T^{\prime}: E^{\prime} \rightarrow L_{p}$ is $p$-decomposable, and there holds

$$
\left\|J S^{\prime}\left(x^{\prime}\right)\right\| \leqq\left\|J T^{\prime}\left(x^{\prime}\right)\right\| \text { for all } x^{\prime} \in E^{\prime} .
$$

From the assumption (4), it follows that $S J^{\prime}$ is $\gamma_{p}$-summing and so is $S=S J^{\prime}$ $P^{\prime}$. By the same way as above, (1) holds.

This completes the proof.

REMARK. In [9], Linde proved that if $S$ and $T$ are bounded linear operators from $L_{p^{\prime}}, 1<p<2$, into a Banach space $E$ such that

$$
\left\|S^{\prime}\left(x^{\prime}\right)\right\|=\left\|T^{\prime}(x)\right\| \text { for all } x^{\prime} \in E^{\prime},
$$


then $T^{\prime}$ is p-decomposable implies $S^{\prime}$ is $p$-decomposable. This result says that in (4) of Theorem 6.3, the inequality can not be replaced by the equality. In fact, it is shown in this case that this property is equivalent to the fact that $E$ is of stable type $p$ and of $S Q_{p}$ type (see Theorem 5.4). On the other hand, Theorem 6.3 is false in the case $p=2$. It is shown that $E$ has one of the properties (2), (3) and (4) for $p=2$ if and only if $E$ is of stable type 2. Finally, we refer to the relation of this reuslt and the works of Linde, Mandrekar and Weron [6] and Thang and Tien [23]. By Lemma 3. 1 and Theorem 6.3, we obtain that $E$ is of stable type $p$ and of $S_{p}$ type, $1<$ $p<2$, if and only if every dual quasi- $p$-nuclear operator from $l_{\mathrm{p}^{\prime}}$ into $E$ is $\gamma_{p}$ Radonifying. This result extends that of Thang and Tien [23], and that of Linde, Mandrekar and Weron [6] for the case $L_{p^{\prime}}=l_{p^{\prime}}$. They proved this result in the case where "quasi-p-nuclear" is replaced by " $p$-summing". Note that every quasi- $p$-nuclear operator is $p$-summing, but in general, the converse is not true.

By a result of Kwapien [4], it follows that a Banach space $E$ is isomorphic to a Hilbert space if and only if every dual 2-summing operator from $l_{2}$ into $E$ is completely summing. In the following, we shall extend this result to the general case $1<p \leqq 2$.

$\mathrm{C}_{\text {OROLlaRY }}$ 6.4. Let $1<p<2$. Then the following properties of a Banach space $E$ are equivalent.

(1) $E$ is of stable type $p$ and of $S_{p}$ type.

(2) For each Banach space $G$ and each $T \in L(G, E), T^{\prime}$ is p-summing implies $T$ is completely summing.

(3) For each (one infinite dimensional) space $L_{p^{\prime}}$ and each $T \in L\left(L_{p^{\prime}}\right.$, $E), T^{\prime}$ is p-summing implies $T$ is completely summing.

PROOF. Since every completely summing operator is $\gamma_{p}$-summing (see Theorem 3.4), the assertion follows from Theorem 6.3.

REMARK. Corollary 6. 4 remains true in the case where " $p$-summing" is replaced by "quasi- $p$-nuclear".

\section{References}

[ 1 ] S. A. ChobANJAN and V. I. TARIElADZE: Gaussian characterizations of certain Banach spaces, J. Multivar. Anal. 7 (1977), 183-203.

[2] S. KWAPIEN : On a theorem of L. Schwartz and its applications to absolutely summing operators, Studia Math. 38 (1970), 193-201.

[ 3 ] S. KWAPIEN : On operators factorizable through $L_{p}$ space, Bull. Soc. Math. France, Mem. 31-32 (1972), 215-225.

[ 4] S. KWAPIEN : Isomorphic characterizations of inner product spaces by orthogonal series with vector valued coefficients, Studia Math. 44 (1972), 583-595. 
[ 5 ] S. KWAPIEN and B. SZYMAŃSKI : Some remarks on Gaussian measures in Banach spaces, Prob. Math. Statist. 1 (1980), 59-65.

[6] W. LINDE, V. MANDREKAR and A. WERON: p-stable measures and p-absolutely summing operators, Lecture Notes in Math. 282, pp. 167-178, Springer 1980.

[ 7] W. Linde, V. I. TARIElADZE and S. A. ChOBANJAN : Characterization of certain classes of Banach spaces by properties of Gaussian measures, Theory Prob. Appl. 25 (1980), 159-164.

[ 8 ] W. LINDE: Operators generating stable measures on Banach spaces, Z. Wahrsch. verw. Gebiete 60 (1982), 171-184.

[ 9 ] W. LINDE: Moments of measures on Banach spaces, Math. Ann. 258 (1982), 277-287.

[10] J. LindENSTRAUSS and A. PELCZYŃSKI : Absolutely summing operators in $L_{p}$-spaces and their applications, studia Math. 29 (1968), 275-326.

[11] J. LindEnSTRAuSS and L. TZAFRIRI : Classical Banach spaces, Lecture Notes in Math. 338, Springer 1973.

[12] V. MANDREKAR and A. WERON : $\alpha$-stable characterization of Banach spaces $(1<\alpha<2)$, J. Multivar. Anal. 11 (1981), 572-580.

[13] B. MAUREY : Espaces de cotype $p, 0<p \leqq 2$, Sém. Maurey-Schwartz, Exp. VII, 1972 $-73$.

[14] B. MAUREY : Théorèmes de factorisation pour les opérateurs linéaires à valeurs dans les espaces $L^{p}$, Asterisque 11, Soc. Math. de France 1974.

[15] D. MouchtaRI: Sur l'existence d'une topologie de type Sazonov sur une espace de Banach, Sém. Maurey-Schwartz, Exp. XVII, 1975-76.

[16] A. PERSSON : On some properties of $p$-nuclear and $p$-integral operators, Studia Math. 33 (1969), 213-222.

[17] A. PERSSON and A. PIETSCH : p-nukleare und $p$-integrale Abbildungen in Banachräumen, Studia Math. 33 (1969), 19-62.

[18] A. PIETSCH : Operator ideals, Berlin: Akademie-Verlag 1978.

[19] L. SchwarTZ: Applications p-sommantes et p-radonifiantes, Sém. Maurey-Schwartz, Exp. IX, 1972-73.

[20] L. SCHWARTZ: Geometry and probability in Banach spaces, Lecture Notes in Math. 852, Springer 1981.

[21] Y. TAKAHASHI and Y. OKAZAKI: Characterizations of subspaces, quotients and subspaces of quotients of $L_{p}$, Hokkaido Math. J. 15 (1986), 233-241.

[22] Y. TAKAHASHI and Y. OKAZAKI: On operators related to $p$-stable measures in Banach spaces, Math. J. Okayama Univ. 27 (1985), 185-195.

[23] D. H. ThAng and N. D. TIEN: Mappings of stable cylindrical measures in Banach spaces, Theory Prob. Appl. 27 (1982), 525-535.

Department of Mathematics

Yamaguchi University

and

Kyushu University 